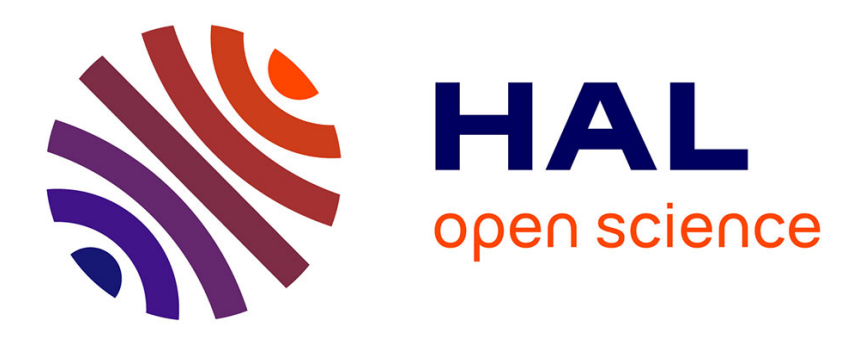

\title{
Choquet integral with stochastic entries
}

Yann Petot, Pierre Vallois, Alexandre Voisin

\section{To cite this version:}

Yann Petot, Pierre Vallois, Alexandre Voisin. Choquet integral with stochastic entries. Fuzzy Sets and Systems, 2021, 412, pp.80-94. 10.1016/j.fss.2020.05.012 . hal-01926695

\section{HAL Id: hal-01926695 \\ https://hal.science/hal-01926695}

Submitted on 19 Nov 2018

HAL is a multi-disciplinary open access archive for the deposit and dissemination of scientific research documents, whether they are published or not. The documents may come from teaching and research institutions in France or abroad, or from public or private research centers.
L'archive ouverte pluridisciplinaire HAL, est destinée au dépôt et à la diffusion de documents scientifiques de niveau recherche, publiés ou non, émanant des établissements d'enseignement et de recherche français ou étrangers, des laboratoires publics ou privés. 


\title{
Choquet integral with stochastic entries
}

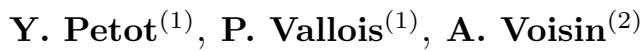

October 19, 2018

(1) Université de Lorraine, Institut de Mathématiques Elie Cartan, INRIA-BIGS, CNRS UMR 7502, BP 239, 54506 Vandœuvre-lès-Nancy Cedex, France

(2) *Université de Lorraine, CNRS, CRAN, F-54000 Nancy, France

\begin{abstract}
We analyse the probabilistic features of the Choquet integral $C_{\mu}(X)$ with respect to a capacity $\mu$ over $\{1, \cdots, n\}$, where the $n$ entries $X=\left(X_{1}, \cdots, X_{n}\right)$ are random variables. Few papers deal with this issue excepted in [8]. We give two different formula for the density function of $C_{\mu}(X)$ when $X_{1}, \cdots, X_{n}$ are i.i.d. random variables. We also calculate the first moment of the Choquet integral and we compare our results with the ones obtained in [8] which mainly concern the cases where the common distribution of $X_{i}$ are either uniform or exponential.
\end{abstract}

Key words and phrases : capacity, Choquet integral, density function, moments, exponential distribution, uniform distribution.

AMS 2000 subject classifications : 60E99, 60K10

\section{Introduction}

Since almost 3 decades, the Choquet integral has become a major "actor" in the field of aggregation function and decision making $[6,7]$. Thanks to theoretical works, many applications have been developed in finance [3], sustainable development [9, 1], image processing [12], recommender system [4], clustering [10], deep-learning [2], to only cite few in recent years.

Despite the amount of studies, the question of uncertainty in the Choquet integral remains under-considered. Such a question is of first importance in many applications and uses. Few works deal with uncertainty considering the entries of the Choquet integral as random variables. Grabisch and Raufaste have proposed in [5] Monte-Carlo simulations to compute in particular cases the empirical mean and standard deviation of the Choquet integral. They draw as conclusion that the exact distribution seems to be hard to obtain. The Choquet integral requires a linear ordering of the entries which generates difficulties when they are random variables. Note that 
ordering a family of probability distributions is usually done using the notion of stochastic dominance [15]. This new notion of order has permitted to Yager in [14] to give an approximation of the mean of the Choquet integral based on the Shapley indices [11]. To circumvent the initial issue, Yager has introduced in [13] a surrogate that allows to compute "the mean-like aggregated value". In both studies, no clear relation is done between the density function of the entries and the density function of the Choquet integral.

As far as we have investigated the literature, the only one contribution which deals with the probability distribution of the Choquet integral has been done by Kojadinovic and Marichal in [8]. Indeed, they have obtained theoretical formulas concerning the moments of the Choquet integral for any form of input distributions. Explicit expressions of the density function of the Choquet integral has also been given but only in the case where the entries are either exponential or uniform.

Let $\mu$ be a capacity on a finite set $S:=\{1, \cdots, n\}$ and $X=\left(X_{1}, \cdots, X_{n}\right)$ be the stochastic entries. In our setting, $X_{1}, \cdots, X_{n}$ are independent random variables. The aim of the paper is to study the Choquet integral $C_{\mu}(X)$ as a random variable. We are able to determine the density of $C_{\mu}(X)$ in Proposition 3.2. The formula is rather complicated but can be simplified (cf Proposition 3.5) if we suppose furthermore that $X_{1}, \cdots, X_{n}$ have the same density, i.e. $X_{1}, \cdots, X_{n}$ are independent and identically distributed (i.i.d.). We have shown a new form of the density of $C_{\mu}(X)$ given in Proposition 3.5 using auxiliary exponential random variables. We provide a general formulation of the law of the Choquet integral which generalizes the result of Kojadinovic and Marichal. These authors has given a non-explicit formula for the moments of order $n$ of the Choquet integral. In the case of the expectation (i.e. $n=1$ ), we obtain an explicit formulation which is in consistence with Proposition 4 in [8] but our proof uses a different way.

Let us briefly explain the structure of the paper. In Section 2, we recall definition of a capacity and the related Choquet integral. In order that the reader has a synthetic overview of our results, the third and fourth sections provides straightforwardly the results for the density function and resp. for the first order moment. Finally, the proofs of the propositions are postponed in Section 5.

\section{Few reminders related to Choquet integral}

We briefly recall the definitions of a capacity and the associated Choquet integral over a finite set $S:=\{1,2, \cdots, n\}$.

Definition 2.1 A capacity, also called a fuzzy measure, $\mu$ over $S$ is a function defined over the family $\mathcal{P}(S)$ of sets included in $S$, valued in $[0,1]$ which is non-decreasing: 


$$
\mu(A) \leq \mu(B), \quad \forall A, B, A \subset B \subset S
$$

and satisfying:

$$
\mu(\emptyset)=0, \quad \mu(S)=1 .
$$

Notation $2.2 \quad$ 1. $\mathcal{S}_{n}$ stands for the group of permutations of $S$.

2. Let $x:=\left(x_{1}, \cdots, x_{n}\right) \in \mathbb{R}^{n}$. There exists $\sigma \in \mathcal{S}_{n}$ such that:

$$
x_{\sigma(1)} \leq \cdots \leq x_{\sigma(n)} .
$$

$\sigma$ is unique if $x_{i} \neq x_{j}$ for all $i \neq j$. Obviously $\sigma$ depends on $x$.

3. It is convenient to adopt the notation: $x_{\sigma(i)}=x_{(i)}$ for any $i \in S$. Therefore (2.2) takes the form:

$$
x_{(1)} \leq \cdots \leq x_{(n)} .
$$

4. If $\sigma: S \rightarrow S$, we set:

$$
\sigma(a: b):=\{\sigma(i), a \leq i \leq b\}, \quad 1 \leq a \leq b \leq n .
$$

We convince that $\sigma(a: b)=\emptyset$ if $b>a$.

Definition 2.3 Let $\mu$ be a capacity over $S$ and $x \in \mathbb{R}^{n}$. The Choquet integral of $x$ with respect to $\mu$ is the real number:

$$
C_{\mu}(x):=\sum_{i=1}^{n} x_{(i)}[\mu(\sigma(i: n))-\mu(\sigma(i+1: n))]
$$

where $\sigma$ is the permutation defined by (2.3).

\section{The law of $C_{\mu}(X)$}

Let $\mu$ be a capacity over $S=\{1, \cdots, n\}$ and $X:=\left(X_{1}, \cdots, X_{n}\right)$ be a random vector. In his Phd-thesis (Contributions aux mesures floues $k$-additives et à l'intégrale de Choquet stochastique. Application aux analyses médice-économiques) $Y$. Petot has considered the case where only one coordinate of $X$ is random. However, We suppose here that all the components $X_{1}, \cdots, X_{n}$ are independent random variables and for any $i \in\{1, \cdots, n\}$ the random variable $X_{i}$ has a density function $f_{i}$.

Let $\mu$ be a given capacity over $S=\{1, \cdots, n\}$. We focus in this section on the density function of the Choquet integral $C_{\mu}(X)$. We calculate it in Proposition 3.2. Unfortunately the formula is not completely explicit since 
it is the sum of multiple Lebesgue integrals which cannot be simplified in the general case. To get a more tractable result, we suppose moreover that $X_{1}, \cdots, X_{n}$ have the same density. In that case, we obtain the simpler formula (3.12). In this identity, it is striking (and unexpected) to see that appear elements of Poisson distributions. Introducing additional exponential random variables, independent of $X$, we can write in Proposition 3.7 the density of $C_{\mu}(X)$ as a sum of functions which can be expressed as expectation of some random variables. This is a new shape of the density function of $C_{\mu}(X)$. Along the presentation, some relation with [8] are proposed to show how both are connected. Proof of the proposition are postponed in Section 5 .

Since the density function of the Choquet integral is complicated, it is natural to wonder if its moments are easier to calculate. We have been only able to (explicitly) calculate the expectation, see Proposition 4.1. We also compare our results to the ones obtained in [8].

Notation 3.1 1. Let $\tau$ be a permutation of $S$.

(a) We set

$$
i_{\tau}:=\max \{1 \leq j \leq n, \mu(\tau(j: n))>0\} .
$$

Since $i \mapsto \mu(\tau(i: n))$ is non-increasing

$$
\begin{aligned}
& \mu(\tau(j: n))=0, \quad j \geq i_{\tau}+1 \\
& \mu(\tau(j: n))>0, \quad 1 \leq j \leq i_{\tau} .
\end{aligned}
$$

(b) We also introduce:

$$
a_{\tau}:=\mu\left(\tau\left(i_{\tau}: n\right)\right)-\mu\left(\tau\left(i_{\tau}+1: n\right)\right)=\mu\left(\tau\left(i_{\tau}: n\right)\right) .
$$

Note that $a_{\tau}>0$.

(c) Let $\theta_{\tau}: \mathbb{R} \rightarrow \mathbb{R}$ be the function:

$\theta_{\tau}(v):=\int_{\mathbb{R}^{n-i_{\tau}}} 1_{\left\{v \leq y_{1} \leq \cdots \leq y_{n-i_{\tau}}\right\}} \prod_{1 \leq j \leq n-i_{\tau}} f_{\tau\left(j+i_{\tau}\right)}\left(y_{j}\right) \prod_{1 \leq j \leq n-i_{\tau}} d y_{j}, \quad v \in \mathbb{R}$.

2. Here, we adher to the conventions:

$$
\int_{\mathbb{R}^{0}}=1, \quad \prod_{i=1}^{0}=1, \quad \sum_{i=1}^{0}=0 .
$$

Proposition 3.2 Let $\phi^{C}$ be the density function of $C_{\mu}(X)$. Then

$$
\phi^{C}(u)=\sum_{\tau \in \mathcal{S}_{n}} \phi_{\tau}(u)
$$


where

$$
\begin{aligned}
\phi_{\tau}(u)= & \frac{1}{a_{\tau}} \int_{\mathbb{R}^{i_{\tau}-1}} f_{\tau\left(i_{\tau}\right)}\left(\frac{u-b_{\tau}}{a_{\tau}}\right) \theta_{\tau}\left(\frac{u-b_{\tau}}{a_{\tau}}\right) \prod_{1 \leq j \leq i_{\tau}-1} f_{\tau(j)}\left(y_{j}\right) \\
& \times 1_{\left\{y_{1} \leq \cdots \leq y_{i_{\tau}-1} \leq \frac{u-b_{\tau}}{a_{\tau}}\right\}} \prod_{1 \leq j \leq i_{\tau}-1} d y_{j}
\end{aligned}
$$

and $b_{\tau}$ is the coefficient which depends on $\left(y_{1}, \cdots, y_{i_{\tau}-1}\right)$ :

$$
b_{\tau}:=\sum_{i=1}^{i_{\tau}-1} y_{i}[\mu(\tau(i: n))-\mu(\tau(i+1: n))] .
$$

For the proof of Proposition 3.2, see Section 5.1. Obviously, Proposition 3.2 is rather complicated. We can explicit the density function $\phi^{C}$ when $n=2$, see Remark CE N'EST PAS LE BON NUMERO 3.6 below. Under additional assumptions we obtain a simplified expression, see Proposition 3.5 below.

Remark 3.3 Let's consider $S=\{1,2\}$ and $S_{2}=\{(1),(2)\}$ where (1) equals the identity and (2) the transposition: $1 \mapsto 2,2 \mapsto 1$. Thus, the function $\phi^{C}$ is the sum of $\phi_{(1)}$ and $\phi_{(2)}$. Let $\Phi_{i}$ be the cumulative distribution function of $X_{i}$.

Case 1.

If $\mu(2)=0$, then $i_{\tau}=1, a_{\tau}=1, b_{\tau}=0$ and

$$
\phi_{(1)}(u)=f_{1}(u)\left(\int_{u}^{+\infty} f_{2}\left(y_{1}\right) d y_{1}\right)=f_{1}(u)\left(1-\Phi_{2}(u)\right) .
$$

If $\mu(\{2\})>0$, then $i_{\tau}=2, a_{\tau}=\mu(2), b_{\tau}=y_{1}(1-\mu(2))$ and

$$
\phi_{(1)}(u)=\frac{1}{\mu(2)}\left(\int_{-\infty}^{u} f_{2}\left(\frac{u-y_{1}(1-\mu(2))}{\mu(2)}\right) f_{1}\left(y_{1}\right) d y_{1}\right) .
$$

Case 2.

If $\mu(1)=0$, then $i_{\tau}=1, a_{\tau}=1, b_{\tau}=0$ and

$$
\phi_{(2)}(u)=f_{2}(u)\left(\int_{u}^{+\infty} f_{1}\left(y_{1}\right) d y_{1}\right)=f_{2}(u)\left(1-\Phi_{1}(u)\right) .
$$

If $\mu(1)>0$, then $i_{\tau}=2, a_{\tau}=\mu(1), b_{\tau}=y_{1}(1-\mu(1))$ and

$$
\phi_{(1)}(u)=\frac{1}{\mu(1)}\left(\int_{-\infty}^{u} f_{1}\left(\frac{u-y_{1}(1-\mu(1))}{\mu(1)}\right) f_{2}\left(y_{1}\right) d y_{1}\right) .
$$

We can go further than Proposition 3.2, in the i.i.d. case. Let $f$ (resp. F) be the common probability density function (resp. cumulative distributive function) of the r.v.'s $X_{i}$. Before stating our result we fix few notations. 
Notation 3.4 Let $\tau$ be a fixed element in $\mathcal{S}_{n}$.

1. We set:

$$
\alpha_{i}:=\mu(\tau(i: n))-\mu(\tau(i+1: n)), \quad 1 \leq i \leq n .
$$

2. Let $l$ be number of elements of $\left\{0 \leq j \leq n, \alpha_{j} \neq 0\right\}$. Note that $\alpha_{i_{\tau}} \neq 0$ and therefore $l \geq 1$. We set:

$$
\left\{1 \leq j \leq n, \alpha_{j} \neq 0\right\}=\left\{j_{1}, \cdots, j_{l}\right\}
$$

where $1 \leq j_{1}<\cdots<j_{l} \leq n$ and

$$
\delta_{i}:=j_{i}-j_{i-1}-1 \quad 2 \leq i \leq l, \quad \delta_{1}=j_{1}-1 .
$$

Note that

$$
i_{\tau}=j_{l}, \quad a_{\tau}=\alpha_{j_{l}} .
$$

3. Let $C_{\tau}$ be the constant:

$$
C_{\tau}:=\frac{1}{a_{\tau}} \frac{1}{\delta_{1} ! \times \cdots \times \delta_{l} !\left(n-j_{l}\right) !} .
$$

Proposition 3.5 We suppose that the rv's $X_{1}, \cdots, X_{n}$ are i.i.d. Then the density function $\phi^{C}$ of $C_{\mu}(X)$ is given by (3.4), where

$$
\begin{aligned}
\phi_{\tau}(u)= & C_{\tau} \int_{\mathbb{R}^{l-1}} f\left(x_{1}\right) \times \cdots \times f\left(x_{l-1}\right) f\left(\frac{u-\widehat{b}_{l-1}}{a_{\tau}}\right)\left(1-F\left(\frac{u-\widehat{b}_{l-1}}{a_{\tau}}\right)\right)^{n-j_{l}} \\
& F\left(x_{1}\right)^{\delta_{1}} \prod_{r=2}^{l-1}\left(F\left(x_{r}\right)-F\left(x_{r-1}\right)\right)^{\delta_{r}} \times\left(F\left(\frac{u-\widehat{b}_{l-1}}{a_{\tau}}\right)-F\left(x_{l-1}\right)\right)^{\delta_{l}} \\
& 1_{\left\{x_{1}<\cdots<x_{l-1}<\frac{u-\widehat{b}_{l-1}}{a_{\tau}}\right\}} d x_{1} \otimes \cdots \otimes d x_{l-1} .
\end{aligned}
$$

and $\widehat{b}_{l-1}:=\sum_{r=1}^{l-1} \alpha_{j_{r}} x_{r}$

Remark 3.6 1. Let $\rho_{1}, \cdots, \rho_{k+1}$ in $[0,+\infty[$. Using the fact that the multiple integral

$$
\begin{aligned}
\int_{\mathbb{R}^{k}} f\left(x_{1}\right) \times \cdots \times f\left(x_{k}\right) F\left(x_{1}\right)^{\rho_{1}} & \prod_{r=2}^{k}\left(F\left(x_{r}\right)-F\left(x_{r-1}\right)\right)^{\rho_{r}} \\
& \times\left(1-F\left(x_{k}\right)\right)^{\rho_{k+1}} d x_{1} \otimes \cdots \otimes d x_{k}
\end{aligned}
$$

equals $\frac{\rho_{1} ! \times \cdots \times \rho_{k+1} !}{\left(k+\rho_{1}+\cdots+\rho_{k+1}\right) !}$ then we can prove that $\int_{\mathbb{R}} \phi_{\tau}(u) d u=\frac{1}{n !}$. 
2. In the uniform case (i.e. when the distribution of all the random variables is uniform over $[0,1])$, the authors in [8] have shown that $\phi^{C}(u)=\sum_{\tau \in \mathcal{S}_{n}} \phi_{\tau}^{*}(u)$, where $\phi_{\tau}^{*}(u)$ can be expressed either as an operator iterated $(n-1)$ times or an integral over $[0,1]^{n-1}$. Since the function $\phi_{\tau}$ defined by (3.12) is an integral over $[0,1]^{l}$, then $\phi_{\tau}^{*}(u)$ and $\phi_{\tau}(u)$ are a priori different. In the case:

$$
\mu(A)<\mu(B), \quad \forall A \subsetneq B \subset S
$$

Kojodinovic and Marichal proved:

$$
\phi^{C}(u)=\frac{1}{n !} \sum_{\tau \in \mathcal{S}_{n}} \sum_{i=0}^{n} \frac{\left(\mu_{i}^{\tau}-u\right)_{+}^{n-1}}{\prod_{j \neq i}\left(\mu_{i}^{\tau}-\mu_{j}^{\tau}\right)}
$$

where $a_{+}$stands for the positive part of a and $\mu_{i}^{\tau}:=\mu(\tau(1, i))$. It is actually possible to recover (3.14) using (3.12) since (3.13) implies $l=n, j_{1}=, \cdots=j_{n}=1, \delta_{1}=\cdots=\delta_{n}=0$.

3. In the exponential case, a nice formula has been given in [8]:

$$
\phi^{C}(u)=\frac{1}{n !} \sum_{\tau \in \mathcal{S}_{n}} \sum_{i=1}^{n} \frac{\left(\mu_{i}^{\tau} / i\right)^{n-2}}{\prod_{j \neq i}\left(\mu_{i}^{\tau} / i-\mu_{j}^{\tau} / j\right)} \exp \left\{-\frac{u}{\left(\mu_{i}^{\tau} / i\right)}\right\}
$$

under the additional assumption

$$
\frac{\mu(A)}{|A|} \neq \frac{\mu(B)}{|B|}, \quad \forall A \neq B
$$

where $|C|$ is the number of elements in $C$.

Proposition 3.5 is a consequence of Proposition 3.2 and its proof is postponed in Section 5.2. Recall that if $Z$ is a random variable which is Poisson distributed with parameter $\lambda$, then

$$
\mathbb{P}(Z=k)=\frac{\lambda^{k}}{k !} e^{-\lambda}, \quad k \in \mathbb{N} .
$$

It is striking to observe that in the right hand-side of (3.12), appears a product of such quantities. Using a Poisson process, we can write (3.12) as the expectation of a random variable.

Let $\left(\xi_{k}\right)_{k \geq 1}$ a sequence of i.i.d. random variables exponentially distributed with parameter 1 , independent of $X_{1}, \cdots, X_{n}$ and

$$
T_{0}:=0, \quad T_{n}:=\xi_{1}+\cdots+\xi_{n} \quad \forall n \geq 1 .
$$

We keep the assumptions given in Proposition 3.5. 
Proposition 3.7 Let $\tau \in \mathcal{S}_{n}$ and $u \in \mathbb{R}$. Then,

$$
\phi_{\tau}(u)=\frac{e}{a_{\tau}} \mathbb{E}\left[f\left(\frac{u-B^{*}}{a_{\tau}}\right) 1_{A_{1} \cap A_{2}(u)}\right]
$$

where

$$
\begin{gathered}
A_{1}:=\left\{T_{n-l} \leq 1<T_{n-l+1}\right\} \bigcap_{r=1}^{l-1}\left\{T_{j_{r}-r} \leq F\left(X_{r}\right)<T_{j_{r}-r+1}\right\} \\
A_{2}(u):=\left\{X_{1}<\cdots<X_{l-1}<\frac{u-B^{*}}{a_{\tau}}\right\} \bigcap\left\{T_{j_{l}-l} \leq F\left(\frac{u-B^{*}}{a_{\tau}}\right)<T_{j_{l}-l+1}\right\} \\
\text { and } B^{*}=: \sum_{r=1}^{l-1} \alpha_{j_{r}} X_{r} .
\end{gathered}
$$

The proof of Proposition 3.7 is given in Section 5.3.

\section{Expectation}

Since the density function of the Choquet integral is complicated, it is natural to wonder if its moments are easier to calculate. We have been only able to (explicitly) calculate the expectation, see Proposition 4.1. We also compare our results to the ones obtained in [8].

We are interested in the caleulation of the expectation and the variance of $G_{\mu}(X)$ where $X$ is a random vector and $\mu$ is a given eapacity.

We set:

$$
\bar{\mu}(k):=\sum_{A \subset S,|A|=k} \mu(A), \quad 0 \leq k \leq n .
$$

where $|A|$ is the number of elements of $A$.

Proposition 4.1 The first moment of $C_{\mu}(X)$ is

$$
\mathbb{E}\left(C_{\mu}(X)\right)=\sum_{i=1}^{n} \bar{\mu}(i) \mathbb{E}\left[F\left(X_{1}\right)^{n-i-1}\left(1-F\left(X_{1}\right)\right)^{i-1} X_{1}\left(n F\left(X_{1}\right)+i-n\right)\right]
$$

The proof of Proposition 4.1 is given in Subsection 5.4.

Remark 4.2 1. In (Proposition 4, [8]), for any integer $r$, an expression of the moment of order $r$ of $C_{\mu}(X)$ has been given but it involves expectations of random variables. In the case $r=1$, the formula coincides with (4.2) The calculation of the second moment of $C_{\mu}(X)$ is possible but is very complicated and do not lead to a simple formula. 
2. In the particular case of uniform distribution, Kojadinovic and Marichal have given an explicit formula for $\mathbb{E}\left[C_{\mu}(X)\right]$ which takes the form

$$
\mathbb{E}\left(C_{\mu}(X)\right)=\frac{1}{(n+1) !} \sum_{i=1}^{n} i !(n-i) ! \bar{\mu}(i)
$$

when $r=1$. It is easy to recover the previous identity from (4.2).

\section{Proofs}

\subsection{Proof of Proposition 3.2}

Let $g: \mathbb{R} \rightarrow \mathbb{R}$ be a test function. Note that if $\tau \in \mathcal{S}_{n}$, then $\{\sigma=\tau\}=$ $\left\{X_{\tau(1)}<\cdots X_{\tau(n)}\right\}$. Therefore,

$$
\mathbb{E}\left[g\left(C_{\mu}(X)\right)\right]=\sum_{\tau \in \mathcal{S}_{n}} \mathbb{E}\left[g\left(C_{\mu}(X)\right) 1_{\left\{X_{\tau(1)} \leq \cdots \leq X_{\tau(n)}\right\}}\right] .
$$

Since the random variables $X_{1}, \cdots, X_{n}$ are independent and $X_{i}$ admits a density function $f_{i}$, we can write the above expectation as:

$\mathbb{E}\left[g\left(C_{\mu}(X)\right)\right]=\sum_{\tau \in \mathcal{S}_{n}} \int_{\mathbb{R}^{n}} g\left(C_{\mu}(x)\right) 1_{\left\{x_{\tau(1)} \leq \cdots \leq x_{\tau(n)}\right\}} f_{1}\left(x_{1}\right) \times \cdots f_{n}\left(x_{n}\right) d x_{1} \otimes \cdots \otimes d x_{n}$

Using the definition of $i_{\tau}$, we have

$$
\begin{aligned}
C_{\mu}(x) & =\sum_{\substack{i=1 \\
i_{\tau}-1}}^{n} x_{\tau(i)}[\mu(\tau(i: n))-\mu(\tau(i+1: n))] \\
& =\sum_{i=1} x_{\tau(i)}[\mu(\tau(i: n))-\mu(\tau(i+1: n))]+x_{\tau\left(i_{\tau}\right)} \mu\left(\tau\left(i_{\tau}: n\right)\right) .
\end{aligned}
$$

In each integral of (5.1), we change the variable $x_{\tau\left(i_{\tau}\right)}$, setting

$$
\begin{aligned}
u & =\sum_{i=1}^{i_{\tau}-1} x_{\tau(i)}[\mu(\tau(i: n))-\mu(\tau(i+1: n))]+x_{\tau\left(i_{\tau}\right)} \mu\left(\tau\left(i_{\tau}: n\right)\right) \\
& =b_{\tau}+a_{\tau} x_{\tau\left(i_{\tau}\right)}
\end{aligned}
$$

where

$$
\begin{aligned}
& a_{\tau}=\mu\left(\tau\left(i_{\tau}: n\right)\right) \\
& b_{\tau}=\sum_{i=1}^{i_{\tau}-1} x_{\tau(i)}[\mu(\tau(i: n))-\mu(\tau(i+1: n))] .
\end{aligned}
$$


Then,

$$
\begin{aligned}
\mathbb{E}\left[g\left(C_{\mu}(X)\right)\right]= & \sum_{\tau \in \mathcal{S}_{n}} \int_{\mathbb{R}} g(u)\left(\frac{1}{a_{\tau}} \int_{\mathbb{R}^{n-1}} f_{\tau\left(i_{\tau}\right)}\left(\frac{u-b_{\tau}}{a_{\tau}}\right) \prod_{j \neq i_{\tau}} f_{\tau(j)}\left(x_{\tau(j)}\right)\right. \\
& \left.\times 1_{\left\{x_{\tau(1)} \leq \cdots \leq x_{\tau\left(i_{\tau}-1\right)} \leq \frac{u-b_{\tau}}{a_{\tau}} \leq x_{\tau\left(i_{\tau}+1\right)} \leq \cdots \leq x_{\tau(n)}\right\}} \prod_{j \neq i_{\tau}} d x_{\tau(j)}\right) d u
\end{aligned}
$$

We can integrate with respect to $d x_{\tau\left(i_{\tau}+1\right)} \otimes \cdots \otimes d x_{\tau(n)}$

$$
\begin{aligned}
\mathbb{E}\left[g\left(C_{\mu}(X)\right)\right]= & \sum_{\substack{\tau \in \mathcal{S}_{n}\\
}} \int_{\mathbb{R}} g(u)\left(\frac{1}{a_{\tau}} \int_{\mathbb{R}^{i_{\tau}-1}} f_{\tau\left(i_{\tau}\right)}\left(\frac{u-b_{\tau}}{a_{\tau}}\right) \theta_{\tau}\left(\frac{u-b_{\tau}}{a_{\tau}}\right)\right. \\
& \times \prod_{\left\{x_{\tau(1)} \leq \cdots \leq x_{\tau\left(i_{\tau}-1\right)} \leq \frac{u-b_{\tau}}{a_{\tau}}\right\}} \prod_{1 \leq j \leq i_{\tau}-1} f_{\tau(j)}\left(x_{\tau(j)}\right) \\
& \left.d x_{\tau(j)}\right) d u
\end{aligned}
$$

with

$$
\theta_{\tau}(v):=\int_{\mathbb{R}^{n-i_{\tau}}} 1_{\left\{v \leq x_{\tau\left(i_{\tau}+1\right)} \leq \cdots \leq x_{\tau(n)}\right\}} \prod_{i_{\tau}+1 \leq j \leq n} f_{\tau(j)}\left(x_{\tau(j)}\right) \prod_{i_{\tau}+1 \leq j \leq n} d x_{\tau(j)} .
$$

This proves (3.5).

\subsection{Proof of Proposition 3.5}

Recall that $\theta_{\tau}(v)$ has been defined by (3.2). This quantity can be calculated explicitly.

Lemma 5.1 For any $v$ :

$$
\theta_{\tau}(v)=\frac{1}{\left(n-i_{\tau}\right) !}(1-F(v))^{n-i_{\tau}} .
$$

Proof. Since the r.v.'s $X_{1}, \cdots, X_{n}$ are i.i.d., they have the same probability density $f$. Therefore $\theta_{\tau}(v)$ takes the simpler form:

$$
\theta_{\tau}(v)=\int_{\mathbb{R}^{m}} f\left(y_{1}\right) \times \cdots \times f\left(y_{m}\right) 1_{\left\{v<y_{1}<\cdots<y_{m}\right\}} d y_{1} \otimes \cdots \otimes d y_{m}
$$

where $m:=n-i_{\tau}$.

Let $g$ be a symmetric function of $m$ variables, i.e. $g\left(y_{1}, \cdots, y_{m}\right)=g\left(y_{\rho(1)}, \cdots, y_{\rho(m)}\right)$ for any permutation $\rho$ of $\{1, \cdots, m\}$ and $\left(y_{1}, \cdots, y_{m}\right) \in \mathbb{R}^{m}$. Then

$$
\int_{\mathbb{R}^{m}} g\left(y_{1}, \cdots, y_{m}\right) d y_{1} \otimes \cdots \otimes d y_{m}=m ! \int_{\mathbb{R}^{m}} g\left(y_{1}, \cdots, y_{m}\right) 1_{\left\{y_{1}<\cdots<y_{m}\right\}} d y_{1} \otimes \cdots \otimes d y_{m} .
$$


Applying this identity with $g\left(y_{1}, \cdots, y_{m}\right)=f\left(y_{1}\right) 1_{\left\{v<y_{1}\right\}} \times \cdots \times f\left(y_{m}\right) 1_{\left\{v<y_{m}\right\}}$ we get:

$$
\begin{aligned}
\theta_{\tau}(v) & =\frac{1}{m !} \int_{\mathbb{R}^{m}} f\left(y_{1}\right) 1_{\left\{v<y_{1}\right\}} \times \cdots \times f\left(y_{m}\right) 1_{\left\{v<y_{m}\right\}} d y_{1} \otimes \cdots \otimes d y_{m} \\
& =\frac{1}{m !}\left(\int_{\mathbb{R}} f(y) 1_{\{v<y\}}\right)^{m} .
\end{aligned}
$$

The result follows since $F(v)=\int_{-\infty}^{v} f(y) d y$.

We are now able to prove Proposition 3.5. By Proposition 3.2, Lemma 5.1 and (3.10), we have:

$$
\begin{aligned}
\phi_{\tau}(u)= & \frac{1}{a_{\tau}\left(n-j_{l}\right) !} \int_{\mathbb{R}^{j_{l}-1}} f\left(\frac{u-b_{\tau}}{a_{\tau}}\right)\left(1-F\left(\frac{u-b_{\tau}}{a_{\tau}}\right)\right)^{n-j_{l}} \\
& \times f\left(y_{1}\right) \times \cdots \times f\left(y_{j_{l}-1}\right) 1_{\left\{y_{1} \leq \cdots \leq y_{j_{l}-1} \leq \frac{u-\tau_{\tau}}{a_{\tau}}\right\}} d y_{1} \otimes \cdots \otimes d y_{j_{l}-1} .
\end{aligned}
$$

By (3.7), (3.8) and (3.10), the coefficient $b_{\tau}$ defined by (3.6) equals

$$
b_{\tau}=\sum_{i=1}^{j_{l}-1} \alpha_{i} y_{i}=\sum_{i=1}^{l-1} \alpha_{j_{i}} y_{j_{i}}
$$

and therefore only depends on the variables $y_{j_{1}} \cdots y_{j_{l-1}}$. Our strategy is to fix $y_{j_{1}} \cdots y_{j_{l-1}}$ and to integrate with respect to the other variables. We integrate first with respect to $d y_{j_{l-1}+1} \otimes \cdots \otimes d y_{j_{l}-1}$ the function $f\left(y_{j_{l-1}+1}\right) \times$ $\cdots \times f\left(y_{j_{l}-1}\right)$ over $\left[y_{j_{l-1}}, \frac{u-b_{\tau}}{a_{\tau}}\right]$ with the constrain $y_{j_{l-1}+1}<\cdots<y_{j_{l}-1}$. Using (5.3), we get :

$$
\frac{1}{\delta_{l} !}\left(F\left(\frac{u-b_{\tau}}{a_{\tau}}\right)-F\left(y_{j_{l-1}}\right)\right)^{\delta_{l}}
$$

which is fixed since $y_{j_{1}}, \cdots, y_{j_{l-1}}$ are supposed to be given. We continue, integrating with respect to $d y_{j_{l-2}+1} \otimes \cdots \otimes d y_{j_{l-1}-1}$ the function $f\left(y_{j_{l-2}+1}\right) \times$ $\cdots f\left(y_{j_{l-1}-1}\right)$ over $\left[y_{j_{l-2}}, y_{j_{l-1}}\right]$ with the constrain $y_{j_{l-2}+1}<\cdots<y_{j_{l-1}-1}$ leads to

$$
\frac{1}{\delta_{l-1} !}\left(F\left(y_{j_{l-1}}\right)-F\left(y_{j_{l-2}}\right)\right)^{\delta_{l-1}} \text {. }
$$

And so on. The last integration is related to $d y_{1} \otimes \cdots \otimes d y_{j_{1}-1}$ and gives

$$
\frac{1}{\left(j_{1}-1\right) !} F\left(y_{j_{1}}\right)^{j_{1}-1}=\frac{1}{\delta_{1} !} F\left(y_{j_{1}}\right)^{\delta_{1}} \text {. }
$$


Finally, setting $x_{1}=y_{j_{1}}, \cdots, x_{l-1}=j_{l-1}$, we get :

$$
\begin{aligned}
\phi_{\tau}(u)= & C_{\tau} \int_{\mathbb{R}^{l-1}} f\left(x_{1}\right) \times \cdots \times f\left(x_{l-1}\right) f\left(\frac{u-\widehat{b}_{l-1}}{a_{\tau}}\right)\left(1-F\left(\frac{u-\widehat{b}_{l-1}}{a_{\tau}}\right)\right)^{n-j_{l}} \\
& \times F\left(x_{1}\right)^{\delta_{1}}\left(\prod_{r=2}^{l-1}\left(F\left(x_{r}\right)-F\left(x_{r-1}\right)\right)^{\delta_{r}}\right)\left(F\left(\frac{u-\widehat{b}_{l-1}}{a_{\tau}}\right)-F\left(x_{l-1}\right)\right)^{\delta_{l}} \\
& \times 1_{\left\{x_{1} \leq \cdots \leq x_{l-1} \leq \frac{u-\widehat{b}_{l-1}}{a_{\tau}}\right\}} d x_{1} \otimes \cdots \otimes d x_{l-1}
\end{aligned}
$$

where:

$$
C_{\tau}=\frac{1}{a_{\tau}\left(n-j_{l}\right) !} \frac{1}{\delta_{1} ! \times \cdots \times \delta_{l} !}
$$

and $\widehat{b}_{l-1}:=\alpha_{j_{1}} x_{1}+\cdots+\alpha_{j_{l-1}} x_{l-1}$.

\subsection{Proof of Proposition 3.7}

According to Proposition 3.5,

$$
\phi_{\tau}(u)=\frac{1}{a_{\tau}} \int_{\mathbb{R}^{l-1}} f\left(x_{1}\right) \times \cdots \times f\left(x_{l-1}\right) \psi_{1}\left(x_{1}, \cdots, x_{l-1}\right) d x_{1} \otimes \cdots \otimes d x_{l-1}
$$

where

$$
\psi_{1}\left(x_{1}, \cdots, x_{l-1}\right):=f\left(\frac{u-\widehat{b}_{l-1}}{a_{\tau}}\right) \psi_{2}\left(x_{1}, \cdots x_{l-1}\right) 1_{\left\{x_{1}<\cdots<x_{l-1}<\frac{u-\widehat{b}_{l-1}}{a_{\tau}}\right\}}
$$

and

$$
\begin{aligned}
\psi_{2}\left(x_{1}, \cdots x_{l-1}\right)= & \frac{F\left(x_{1}\right)^{\delta_{1}}}{\delta_{1} !} \prod_{r=2}^{l-1} \frac{\left(F\left(x_{r}\right)-F\left(x_{r-1}\right)\right)^{\delta_{r}}}{\delta_{r} !} \\
& \times \frac{\left(F\left(\frac{u-\widehat{b}_{l-1}}{a_{\tau}}\right)-F\left(x_{l-1}\right)\right)^{\delta_{l}}}{\delta_{l} !} \frac{\left(1-F\left(\frac{u-\widehat{b}_{l-1}}{a_{\tau}}\right)\right)^{n-j_{l}}}{\left(n-j_{l}\right) !}
\end{aligned}
$$

It is very convenient to introduce:

$$
y_{0}:=0, y_{i}:=F\left(x_{i}\right) \forall 1 \leq i \leq l-1, y_{l}:=F\left(\frac{u-\widehat{b}_{l-1}}{a_{\tau}}\right), y_{l+1}:=1 .
$$

Since $x_{1}<\cdots<x_{l-1}<\frac{u-\widehat{b}_{l-1}}{a_{\tau}}$, then $y_{0} \leq \cdots<y_{l+1}$ and

$$
\psi_{2}\left(x_{1}, \cdots, x_{l-1}\right)=e \prod_{r=1}^{l+1} \frac{\left(y_{r}-y_{r-1}\right)^{\delta_{r}}}{\delta_{r} !} e^{-\left(y_{r}-y_{r-1}\right)} .
$$

where $\delta_{l+1}:=n-j_{l}$.

Let $\left(N_{t}\right)_{t \geq 0}$ be a Poisson process with parameter 1 and independent of $X_{1}, \cdots, X_{n}$. Then 
1. The random variables $N_{y_{1}}, N_{y_{2}}-N_{y_{1}}, \cdots, N_{y_{l+1}}-N_{y_{l}}$ are independent,

2. for any $r \in\{1, \cdots, l+1\}$, the random variable $N_{y_{r}}-N_{y_{r-1}}$ is Poisson distributed with parameter $y_{r}-y_{r-1}$.

We are now able to interpret $\psi_{2}\left(x_{1}, \cdots x_{l-1}\right)$ :

$$
\begin{aligned}
\psi_{2}\left(x_{1}, \cdots, x_{l-1}\right) & =e \prod_{r=1}^{l+1} \mathbb{P}\left(N_{y_{r}}-N_{y_{r-1}}=\delta_{r}\right) \\
& =e \mathbb{P}\left(\bigcap_{r=1}^{l+1}\left\{N_{y_{r}}-N_{y_{r-1}}=\delta_{r}\right\}\right) \\
& =e \mathbb{P}\left(\bigcap_{r=1}^{l+1}\left\{N_{y_{r}}=\delta_{1}+\cdots+\delta_{r}\right\}\right)
\end{aligned}
$$

Let $\left(T_{n}\right)_{n \geq 1}$ be the increasing sequence of the jump times of $\left(N_{t}\right)_{t \geq 0}$ :

$$
N_{t}=\sum_{n \geq 1} 1_{\left\{T_{n} \leq t\right\}}, \quad t \geq 0 .
$$

Consequently:

$$
\left\{N_{t}=k\right\}=\left\{T_{k} \leq t<T_{k+1}\right\}, \quad t \geq 0, k \in \mathbb{N}
$$

and therefore

$$
\psi_{2}\left(x_{1}, \cdots, x_{l-1}\right)=e \mathbb{P}\left(\bigcap_{r=1}^{l+1}\left\{T_{j_{r}-r} \leq y_{r}<T_{j_{r}-r+1}\right\}\right)
$$

because relation (3.9) implies:

$$
\delta_{1}+\cdots+\delta_{r}=j_{r}-r, \quad 1 \leq r \leq l+1
$$

with the convention $j_{l+1}=n+1$.

It is well-know that $\left(T_{n}\right)_{n \geq 1}$ is distributed as $\left(\xi_{1}+\cdots+\xi_{n}\right)_{n \geq 1}$ where $\left(\xi_{k}\right)_{k \geq 1}$ is a sequence of i.i.d. random variables exponentially distributed with parameter 1. Finally relation (5.4) and the independency of $\left(\xi_{k}\right)_{k \geq 1}$ and $\left(X_{1}, \cdots, X_{n}\right)$ implies (3.18).

\subsection{Proof of Proposition 4.1}

Recall that the quantity $\bar{\mu}(k)$ has been defined by (4.1).

Lemma 5.2 Let $1 \leq i \leq n$. Then

$$
\sum_{\tau \in \mathcal{S}_{n}} \mu(\tau(i: n))=(i-1) !(n-i+1) ! \bar{\mu}(n-i+1) .
$$


Proof. We have:

$$
\begin{aligned}
\sum_{\tau \in \mathcal{S}_{n}} \mu(\tau(i: n)) & =\sum_{A \subset S,|A|=n-i+1}\left(\sum_{\tau \in \mathcal{S}_{n}, A=\tau(i: n)} \mu(\tau(i: n))\right) \\
& =\sum_{A \subset S,|A|=n-i+1} \mu(A)\left(\sum_{\tau \in S, A=\tau(i: n)} 1\right) \\
& =(i-1) !(n-i+1) ! \sum_{A \subset S,|A|=n-i+1} \mu(A)
\end{aligned}
$$

since if $A \subset S$ is given and $A=\tau(i: n)$ then the image of $\{1, \cdots, i-1\}$ (resp. $\{i, \cdots, n\})$ is $A^{c}$ (resp. $A$ ) and the number of possibilities is $(i-1)$ !× $(n-i+1)$ !. Then $(5.5)$ is a direct consequence of (4.1).

Lemma 5.3 For any $1 \leq i \leq n$,

$$
\mathbb{E}\left(X_{i} 1_{\left\{X_{1}<\cdots<X_{n}\right\}}\right)=\frac{1}{(i-1) !(n-i) !} \mathbb{E}\left(F\left(X_{1}\right)^{i-1}\left(1-F\left(X_{1}\right)\right)^{n-i} X_{1}\right) .
$$

Proof. Let $1 \leq i \leq n$, then

$$
\mathbb{E}\left(X_{i} 1_{\left\{X_{1}<\cdots<X_{n}\right\}}\right)=\mathbb{E}\left(1_{\left\{X_{1}<\cdots<X_{i-1}<X_{i}\right\}} X_{i} 1_{\left\{X_{i}<X_{i+1} \cdots<X_{n}\right\}}\right) .
$$

Recall that $X_{1}, \cdots, X_{n}$ are i.i.d. then

$$
\mathbb{E}\left(X_{i} 1_{\left\{X_{1}<\cdots<X_{n}\right\}}\right)=\mathbb{E}\left(\theta_{i-1}\left(X_{i}\right) X_{i} \theta^{n-i}\left(X_{i}\right)\right)
$$

where

$$
\begin{aligned}
\theta_{k}(x) & :=\int_{\mathbb{R}^{k}} f\left(x_{1}\right) \times \cdots \times f\left(x_{k}\right) 1_{\left\{x_{1}<\cdots<x_{k}<x\right\}} d x_{1} \otimes \cdots \otimes d x_{k} \\
\theta^{k}(x) & :=\int_{\mathbb{R}^{k}} f\left(x_{1}\right) \times \cdots \times f\left(x_{k}\right) 1_{\left\{x<x_{1}<\cdots<x_{k}\right\}} d x_{1} \otimes \cdots \otimes d x_{k} .
\end{aligned}
$$

Using (5.3), $\theta_{k}(x)$ can be simplified:

$$
\begin{aligned}
\theta_{k}(x) & =\frac{1}{k !} \int_{\mathbb{R}^{k}} f\left(x_{1}\right) \times \cdots \times f\left(x_{k}\right) 1_{\left\{x_{1}<x, \cdots,<x_{k}<x\right\}} d x_{1} \otimes \cdots \otimes d x_{k} \\
& =\frac{F(x)^{k}}{k !} .
\end{aligned}
$$

Similarly,

$$
\theta^{k}(x)=\frac{(1-F(x))^{k}}{k !} .
$$

The proof of (5.6) is now complete since $X_{i}$ and $X_{1}$ have the same distribution. 
We are now able to prove Proposition 4.1.

By definition,

$$
C_{\mu}(X)=\sum_{i=1}^{n} X_{\sigma(i)}[\mu(\sigma(i: n))-\mu(\sigma(i+1: n))]
$$

where $\sigma$ is the random permutation such that $X_{\sigma(1)}<\cdots<X_{\sigma(n)}$. We modify the previous sum:

$$
\begin{aligned}
C_{\mu}(X) & =\sum_{i=1}^{n} X_{\sigma(i)} \mu(\sigma(i: n))-\sum_{i=1}^{n} X_{\sigma(i)} \mu(\sigma(i+1: n)) \\
& =\sum_{i=1}^{n} \mu(\sigma(i: n))\left(X_{\sigma(i)}-X_{\sigma(i-1)}\right)
\end{aligned}
$$

with the conventions: $\sigma(0)=0, X_{0}=0$ and $\mu(\sigma(n+1: n))=0$. Therefore

$$
\begin{aligned}
C_{\mu}(X) & =\sum_{\tau \in \mathcal{S}_{n}}\left(\sum_{i=1}^{n} \mu(\sigma(i: n))\left(X_{\sigma(i)}-X_{\sigma(i-1)}\right)\right) 1_{\{\sigma=\tau\}} \\
& \left.=\sum_{\tau \in \mathcal{S}_{n}}\left(\sum_{i=1}^{n} \mu(\tau(i: n))\left(X_{\tau(i)}-X_{\tau(i-1)}\right)\right) 1_{\left\{X_{\tau(1)}<\cdots<X_{\tau(n)}(5)\right\}}\right)
\end{aligned}
$$

We take the expectation and we use the crucial fact that $\left(X_{\tau(1)}, \cdots, X_{\tau(n)}\right)$ is distributed as $\left(X_{1}, \cdots, X_{n}\right)$ :

$$
\begin{aligned}
\mathbb{E}\left(C_{\mu}(X)\right) & =\sum_{\tau \in \mathcal{S}_{n}}\left(\sum_{i=1}^{n} \mu(\tau(i: n)) \mathbb{E}\left[\left(X_{\tau(i)}-X_{\tau(i-1)}\right) 1_{\left\{X_{\tau(1)}<\cdots<X_{\tau(n)}\right\}}\right]\right) \\
& =\sum_{\tau \in \mathcal{S}_{n}}\left(\sum_{i=1}^{n} \mu(\tau(i: n)) \mathbb{E}\left[\left(X_{i}-X_{i-1}\right) 1_{\left\{X_{1}<\cdots<X_{n}\right\}}\right]\right) \\
& =\sum_{i=1}^{n}\left(\sum_{\tau \in \mathcal{S}_{n}} \mu(\tau(i: n))\right) \mathbb{E}\left[\left(X_{i}-X_{i-1}\right) 1_{\left\{X_{1}<\cdots<X_{n}\right\}}\right] .
\end{aligned}
$$

Using Lemma 5.3, we gat for $i \geq 2$ :

$$
\begin{aligned}
\mathbb{E}\left[\left(X_{i}-X_{i-1}\right) 1_{\left\{X_{1}<\cdots<X_{n}\right\}}\right]= & \mathbb{E}\left[X_{i} 1_{\left\{X_{1}<\cdots<X_{n}\right\}}\right]-\mathbb{E}\left[X_{i-1} 1_{\left\{X_{1}<\cdots<X_{n}\right\}}\right] \\
= & \frac{1}{(i-1) !(n-i) !} \mathbb{E}\left(F\left(X_{1}\right)^{i-1}\left(1-F\left(X_{1}\right)\right)^{n-i} X_{1}\right) \\
& -\frac{1}{(i-2) !(n-i+1) !} \mathbb{E}\left(F\left(X_{1}\right)^{i-2}\left(1-F\left(X_{1}\right)\right)^{n-i+1}\right. \\
& \left.\times X_{1}\right) .
\end{aligned}
$$

Consequently

$\mathbb{E}\left[\left(X_{i}-X_{i-1}\right) 1_{\left\{X_{1}<\cdots<X_{n}\right\}}\right]=\frac{1}{(i-1) !(n-i+1) !} \mathbb{E}\left(F\left(X_{1}\right)^{i-2}\left(1-F\left(X_{1}\right)\right)^{n-i} X_{1} Y\right)$ 
where

$$
\begin{aligned}
Y & :=(n-i+1) F\left(X_{1}\right)-(i-1)\left(1-F\left(X_{1}\right)\right. \\
& =n F\left(X_{1}\right)-i+1 .
\end{aligned}
$$

We use finally Lemma 5.2:

$\mathbb{E}\left(C_{\mu}(X)\right)=\sum_{i=1}^{n} \bar{\mu}(n-i+1) \mathbb{E}\left(F\left(X_{1}\right)^{i-2}\left(1-F\left(X_{1}\right)\right)^{n-i} X_{1}\left(n F\left(X_{1}\right)-i+1\right)\right)$.

ReferencesReferences

\section{References}

[1] Glin Bykzkan, Orhan Feyzio?lu, and Fethullah Ger. Selection of sustainable urban transportation alternatives using an integrated intuitionistic fuzzy choquet integral approach. Transportation Research Part D: Transport and Environment, 58:186 - 207, 2018.

[2] Camila Alves Dias, Jéssica C. S. Bueno, Eduardo N. Borges, Silvia S. C. Botelho, Graçaliz Pereira Dimuro, Giancarlo Lucca, Javier Fernandéz, Humberto Bustince, and Paulo Lilles Jorge Drews Junior. Using the choquet integral in the pooling layer in deep learning networks. In Guilherme A. Barreto and Ricardo Coelho, editors, Fuzzy Information Processing, pages 144-154, Cham, 2018. Springer International Publishing.

[3] Joo J.M. Ferreira, Marjan S. Jalali, and Fernando A.F. Ferreira. Enhancing the decision-making virtuous cycle of ethical banking practices using the choquet integral. Journal of Business Research, 88:492 - 497, 2018 .

[4] Soumana Fomba, Pascale ZARATÉ, and Marc Kilgour. A Recommender system based on MultiCriteria Aggregation. In 2nd International Conference on Decision Support Systems TechnologY (ICDSST 2016) EWGpages pp. 1-7, Plymouth, United Kingdom, May 2016.

[5] M. Grabisch and E. Raufaste. An empirical study of statistical properties of the choquet and sugeno integrals. IEEE Transactions on Fuzzy Systems, 16(4):839-850, Aug 2008.

[6] Michel Grabisch and Christophe Labreuche. A decade of application of the choquet and sugeno integrals in multi-criteria decision aid. 4OR, 6(1):1-44, Mar 2008.

[7] Michel Grabisch and Christophe Labreuche. A decade of application of the Choquet and Sugeno integrals in multi-criteria decision aid. Annals of Operations Research, 175(1):247-290, March 2010. 
[8] Ivan Kojadinovic and Jean-Luc Marichal. On the moments and distribution of discrete choquet integrals from continuous distributions. Journal of Computational and Applied Mathematics, 230(1):83 - 94, 2009.

[9] Myriam Merad, Nicolas Dechy, Lisa Serir, Michel Grabisch, and Frédéric Marcel. Using a multi-criteria decision aid methodology to implement sustainable development principles within an Organization. European Journal of Operational Research, pages 603-613, 2013.

[10] Andre G.C. Pacheco and Renato A. Krohling. Aggregation of neural classifiers using choquet integral with respect to a fuzzy measure. Neurocomputing, 292:151 - 164, 2018.

[11] L. S. Shapleyd. A value for n-person games. In H.W.Kuhn andA.W. Tucker, editor, Contributions to Game Theory, pages 307-317. Eds. Princeton, NJ, USA: Princeton Univ. Press, 1953.

[12] Soumaya Trabelsi Ben Ameur, Florence Cloppet, Sellami Dorra, and Laurent Wendling. Choquet Integral based Feature Selection for Early Breast Cancer Diagnosis from MRIs. In ICPRAM, volume Proceedings of the 5th International Conference on Pattern Recognition Applications and Methods, pages 351 - 358, Rome, Italy, February 2016.

[13] R. R. Yager. Evaluating choquet integrals whose arguments are probability distributions. IEEE Transactions on Fuzzy Systems, 24(4):957965, Aug 2016.

[14] R. R. Yager. On using the shapley value to approximate the choquet integral in cases of uncertain arguments. IEEE Transactions on Fuzzy Systems, 26(3):1303-1310, June 2018.

[15] Ronald R. Yager. Stochastic dominance for measure based uncertain decision making. International Journal of Intelligent Systems, 29(10):881905, 2014. 